\title{
Undiagnosed Obstructive Sleep Apnea and Acute COVID-19 Infection-A Case Series
}

\author{
Hyeon Hui Kang, Jin Hyoung Kim, Byung Ju Kang, Taehoon Lee, \\ Seung Won Ra, Kwang Won Seo, Yangjin Jegal, and Jong Joon Ahn \\ Division of Pulmonary, Critical Care and Sleep Medicine, Department of Internal Medicine, \\ Ulsan University Hospital, University of Ulsan College of Medicine, Ulsan, Korea
}

\begin{abstract}
A recently published study on coronavirus disease 2019 (COVID-19) and obstructive sleep apnea (OSA) suggested that there might be an association between certain risk factors and comorbidities associated with OSA, which are also associated with poor COVID-19 outcomes. However, it is unclear whether undiagnosed OSA correlates with COVID-19 severity in a South Korean population. We identified 7 patients who presented with nocturnal hypoxemia during hospitalization due to COVID-19. All patients underwent polysomnography 5-9 weeks after the infection. We retrospectively collected the patients' baseline characteristics, hospital admission data, and polysomnography findings. Of the 7 patients, all were diagnosed with OSA after COVID-19 infection. Their mean $( \pm S D)$ age was $45.4 \pm 16.3$ years, $57.1 \%$ were men, and their mean $\left( \pm\right.$ SD) body mass index was $33.4 \pm 6.0 \mathrm{~kg} / \mathrm{m}^{2}$. Six patients presented with COVID-19-related pneumonia on chest X-rays, 3 of whom were admitted to the intensive care unit during the acute phase. The overnight polysomnography showed a mean AHI of 59.0 $\pm 38.5 / \mathrm{h}$ and an oxygen desaturation index of $57.6 \pm 39.7 / \mathrm{h}$. Undiagnosed OSA is a prevalent condition associated with moderate to severe COVID-19 infection. The study patients with sleep apnea and COVID-19 had obesity and severe oxygen desaturation but did not complain of daytime sleepiness.
\end{abstract}

Keywords: COVID-19; Sleep apnea; Polysomnography

Received: December 10, 2021 Revised: December 11, 2021 Accepted: December 12, 2021

Corresponding author: Hyeon Hui Kang, MD, PhD, Division of Pulmonary, Critical Care and Sleep Medicine, Department of Internal Medicine, Ulsan University Hospital, University of Ulsan College of Medicine, 877 Bangeojinsunhwando-ro, Dong-gu, Ulsan 44033, Korea.

Tel: 82-52-250-8660, Fax: 82-52-250-7048, E-mail: khh3822@naver.com

(a) This is an Open Access article distributed under the terms of the Creative Commons Attribution Non-Commercial License (https://creativecommons.org/licenses/by$\mathrm{nc/4.0)}$ which permits unrestricted non-commercial use, distribution, and reproduction in any medium, provided the original work is properly cited.

\section{INTRODUCTION}

Coronavirus disease 2019 (COVID-19) has severely affected healthcare systems worldwide. Although age, hypertension, cardiovascular disease, lung disease, and diabetes mellitus are apparently the main risk factors for poorer outcomes in COVID-19 [1], a possible role has also been ascribed to sleep-disordered breathing (SDB) [2-4]. A recent preliminary study that collected questionnaire data in a case series of COVID-19 pneumonia showed that $25 \%$ of patients presented a history of SDB [5]. In recent months, a number of studies have suggested that the presence of obstructive sleep apnea (OSA) as a comorbidity in patients with COVID-19 increases the risk of hospitalization and mortality [6,7].

A study reported that OSA is a risk factor of COVID-19 in South Korea [8]. In an analysis of the National Health Insurance Service (NHIS)-COVID-19 cohort, OSA was associated with a 1.65-fold higher incidence of COVID-19 than in the control group. However, this was the only study in South Korea to mention the association between COVID-19 and SDB, and there have been no studies on the clinical characteristics and prognosis of patients with sleep apnea and COVID-19 in South Korea. Due to the limitations of the NHIS data, there have been few reports describing patients admitted to the hospital with COVID-19 in South Korea. A better characterization of COVID-19 infection in patients with SDB is important for understanding the clinical course and outcomes of COVID-19 and determining the critical care capacity and decision-making process for therapeutic interventions. The aim of our report is to describe the demographic characteristics, coexisting conditions, imaging findings, and outcomes of patients with SDB and COVID-19 in South Korea. 


\section{METHODS}

Participants and study design

We identified patients 18 years of age or older with laboratoryconfirmed COVID-19 infections who were admitted to Ulsan University Hospital between October 2020 and June 2021. A confirmed case of COVID-19 was defined as a positive result on a reverse-transcription polymerase-chain-reaction (RT-PCR) assay of a specimen collected on a nasopharyngeal swab or sputum. Given that our hospital is the only base hospital for COVID-19 treatment in Ulsan, all patients in Ulsan who require hospitalization are admitted to our hospital. If patients with COVID-19 pneumonia showed clinical and radiological improvement during hospitalization but showed hypoxemia in pulse oximetry during sleep, they underwent elective laboratory polysomnography 5-9 weeks after the COVID-19 infection.

The Institutional Review Board at Ulsan University Hospital independently approved the study (UUH 2021-11-040). Informed consent was waived, and we retrospectively analyzed only deidentified data. We obtained demographic data, information on clinical symptoms or signs at presentation, and radiological results during hospitalization from the electronic medical records. All radiological assessments were performed at the discretion of the treating physician.

Participants completed a standard polysomnography (Comet Plus, Astro-Med, Inc., West Warwick, RI, USA), which included electroencephalography, electrooculography, electromyography, electrocardiography, pulse oximetry for oxygen saturation, and a microphone to detect snoring. The airflow was measured using an oronasal thermistor and a nasal pressure transducer, and the respiratory effort of thoracoabdominal movement was measured using respiratory inductance plethysmography. Sleep and associated events were scored according to the American Academy of Sleep Medicine manual $[9,10]$. The apnea-hypopnea index (AHI) was defined as the mean number of episodes of apnea and hypopnea per hour of total sleep time. Apnea was defined as a decrease in the peak thermal sensor excursion by at least $90 \%$ from baseline for at least 10 seconds. A hypopnea episode was defined as a decrease in the nasal pressure signal by at least $30 \%$ from baseline for at least 10 seconds, with a reduction in oxygen saturation of at least $4 \%$ from the pre-event baseline. The patients completed the following sleep-related questionnaires: Pittsburgh Sleep Quality Index; Epworth sleepiness scale; and Berlin questionnaire.

\section{Statistical analysis}

Descriptive statistics were employed to summarize the data, and the results are reported as medians and interquartile ranges or means and standard deviations, as appropriate. The categorical variables are summarized as counts and percentages. No imputation was performed for missing data.

\section{RESULTS}

We identified 7 patients ( 4 men, 3 women) with nighttime hypoxemia during hospitalization, and all patients had confirmed OSA after COVID-19 treatment. The baseline characteristics of all participants are shown in Table 1. The participants' mean age was $45.4 \pm 16.3$ years, their mean body mass index was $33.4 \pm 6.0$ $\mathrm{kg} / \mathrm{m}^{2}$, and the prevalence of smoking was $28.6 \%$. The common comorbidities were hypertension and hyperlipidemia. None of the patients had diagnosed coronary heart disease or stroke. Six patients presented COVID-19-related pneumonia in the chest $\mathrm{X}$-rays and underwent oxygen therapy in the medical ward or intensive care unit (ICU). Three patients were hospitalized in the ICU during the acute phase, with a mean ICU stay of 11 days. In the ICU, all patients received oxygen by high-flow nasal cannula; however, we did not administer continuous positive airway pressure (CPAP) or bilevel positive airway pressure therapy. All pa-

Table 1. Clinical characteristics and sleep assessment of the participants

\begin{tabular}{|c|c|}
\hline Characteristics & Values $(\mathrm{n}=7)$ \\
\hline Age, years (range) & $45.4 \pm 16.3(27-67)$ \\
\hline Male & $4(57.1)$ \\
\hline $\mathrm{BMI}, \mathrm{kg} / \mathrm{m}^{2}$ & $33.4 \pm 6.0$ \\
\hline \multicolumn{2}{|l|}{ Tobacco } \\
\hline Nonsmoker & $5(71.4)$ \\
\hline Current & $2(28.6)$ \\
\hline \multicolumn{2}{|l|}{ Comorbidities } \\
\hline Hypertension & $3(42.6)$ \\
\hline Diabetes mellitus & $1(14.3)$ \\
\hline Hyperlipidemia & $3(42.6)$ \\
\hline Lowest $\mathrm{SpO}_{2}(\%)$ on admission & $90.0 \pm 3.4$ \\
\hline \multicolumn{2}{|l|}{ Chest radiography findings } \\
\hline Normal & $1(14.3)$ \\
\hline Bilateral ground glass opacities & $2(28.6)$ \\
\hline Bilateral consolidation & $4(57.1)$ \\
\hline Need for oxygen treatment & $6(85.7)$ \\
\hline Nasal prong & $3(42.6)$ \\
\hline High-flow nasal cannula & $3(42.6)$ \\
\hline ICU admission & $3(42.6)$ \\
\hline Days in the ICU (range) & $11.3 \pm 3.1(8-14)$ \\
\hline \multicolumn{2}{|l|}{ Sleep questionnaire } \\
\hline Pittsburgh sleep quality index & $6.6 \pm 4.0$ \\
\hline Epworth sleepiness scale & $4.3 \pm 3.3$ \\
\hline Berlin questionnaire, high risk & $4(57.1)$ \\
\hline \multicolumn{2}{|l|}{ Polysomnographic findings } \\
\hline Sleep efficiency (\%) & $79.8 \pm 12.4$ \\
\hline Apnea-hypopnea index (/h) & $59.0 \pm 38.5$ \\
\hline Minimum oxygen saturation (\%) & $77.9 \pm 9.8$ \\
\hline Oxygen desaturation index, $\geq 3 \%(/ \mathrm{h})$ & $57.6 \pm 39.7$ \\
\hline Continuous positive airway pressure $\left(\mathrm{cm} \mathrm{H}_{2} \mathrm{O}\right)$ & $9.3 \pm 1.4$ \\
\hline
\end{tabular}

Values are given as the mean \pm standard deviation or number (\%), unless otherwise indicated. BMI, body mass index; $\mathrm{SpO}_{2}$, peripheral oxygen saturation; ICU, intensive care unit 
tients were discharged without supplementary oxygen.

We documented a mean follow-up time of 7.7 weeks (range 5-9 weeks) after COVID-19 infection. The laboratory polysomnography showed a mean AHI of $59.0 \pm 38.5 / \mathrm{h}$ and an oxygen desaturation index of $57.6 \pm 39.7 / \mathrm{h}$. The mean lowest oxygen saturation was $77.9 \% \pm 9.8 \%$. Regarding the sleep questionnaires, we found no significant subjective sleepiness according to the Epworth sleepiness scale questionnaire. Four of the patients (57.1\%) showed a high-risk of OSA according to the Berlin questionnaire. Of the 7 patients, 6 underwent CPAP titration. The mean positive airway pressure was $9.3 \mathrm{~cm} \mathrm{H}_{2} \mathrm{O}$.

\section{DISCUSSION}

This case series describes 7 patients diagnosed with SDB with nocturnal hypoxemia and laboratory-confirmed COVID-19 infection. According to the current literature, this is the first description aimed at evaluating the association between undiagnosed SDB and COVID-19 outcomes in South Korea. Our findings support the association between SDB and poorer COVID-19 outcomes.

The patients in our series presented with moderate to severe COVID-19 infection. COVID-19 severity was defined based on the World Health Organization's Clinical Progression Scale: 1) mild disease-outpatient care; 2) moderate disease-hospitalization; and 3) severe disease-intensive care or death [11]. All patients were admitted to our hospital, and 6 patients presented with COVID-19-related hypoxemia and pneumonia. Among those who required oxygen therapy, 3 underwent high-flow oxygen therapy up to a fraction of inspired oxygen of $70 \%$.

Although our study is a retrospective analysis, it can be of clinical significance in suggesting that patients with severe COVID-19 who do not have significant subjective sleepiness or those who have not been assigned to a high-risk group in the Berlin questionnaire are likely to have undiagnosed sleep apnea. Previous studies evaluating the association between SDB and COVID-19 have suggested this association. In the CORONADO observational study, treated SDB was reported to be an independent risk of mortality after 7 days, with an odds ratio (OR) of 2.8 (confidence interval [CI], 1.46-5.38) [12]. In another study that included 46 patients hospitalized due to COVID-19, sleep apnea was diagnosed in $75 \%$ of the sample [13]. In an observational study in the United States, patients with SDB had an OR of 1.53 (CI, 1.09-2.15) for mortality and an OR of 1.29 (CI, 1.03-1.62) for ICU admission [6]. The plausible mechanisms for these associations include systemic and chronic inflammation, which are commonly observed in patients with untreated SDB. Published studies have suggested that when controlling for obesity, the presence of OSA is associated with decreased pulmonary function, decreased lungtransfer factor for carbon monoxide, and increased lung inflammation [14]. These conditions might therefore explain, at least in part, why patients with OSA are generally at increased risk for pneumonia [15]. These observations might therefore provide for the possibility of increased disease severity in the COVID-19 setting. In this context, the pathophysiology associated with untreated OSA might not only present a predisposing factor for developing severe or critical illness in COVID-19 but also, once infection has occurred, repeated airway obstruction with the generation of negative intrathoracic pressure and associated shear forces that can lead to worsening lung injury [16].

In this study, we found that 7 patients with undiagnosed sleep apnea presented with very high body mass and oxygen desaturation indices compared with the patients with sleep apnea generally treated at sleep clinics in South Korea. Although it is difficult to generalize due to the small number of samples, this finding suggests that patients with obesity and hypoxemia during sleep, even after recovery from moderate to severe COVID-19, are more likely to have undiagnosed sleep apnea.

The management of patients with OSA in sleep centers includes diagnostic procedures such as polysomnography and treatment with positive air pressure. Given the risk of spreading the virus in both of these procedures, many sleep centers in Western countries have suspended routine procedures, limiting activity to urgent cases. In the early days of the pandemic, numerous national sleep medicine societies provided guidelines for implementing these infection control strategies in sleep centers. An early mitigation strategy document from the authoritative American Academy of Sleep Medicine strongly recommended postponing nonurgent procedures [17], recommendations that were confirmed worldwide [18-21]. For many months, patients with suspected OSA, who usually have waited a varying amount of time from the first triage visit to treatment, therefore had to endure a further delay in getting treated.

The situation for sleep medicine in South Korea is not much different, with delays in the diagnosis and treatment of patients with sleep apnea given the rise in the number of centers postponing polysomnography in the early stages of the pandemic or conducting polysomnography only if the COVID-19 test were negative. Given that the first recommendations from the sleep societies were from last year, updated protocols are needed. In this context, we provide an example for the clinical suspicion and diagnosis of sleep apnea.

A potential contributor to the high morbidity among COVID-19 patients with obesity might be the high prevalence of undiagnosed OSA. Although it is difficult to actively diagnose sleep apnea in the current pandemic situation, attention needs to be paid as to whether a COVID-19 patient with moderate or higher severity has been underdiagnosed for this disease.

The study's main limitations are related to the small sample size; however, this study provides evidence regarding the association between undiagnosed OSA and acute COVID-19 in a South Korean population. This study also has an inclusion bias as a methodological limitation, and the conclusions are restricted to the data obtained from the included patients. While taking all these issues into account, we believe that the results of this study can provide an insight into the association between OSA and COV- 
ID-19 in South Korea. Although COVID-19 severity can vary among individuals, the disease can have poor outcomes, and as the pandemic progresses, medical resources are being depleted. It is therefore important to identify the risk factors, as this can be beneficial for high-risk patients. Further studies are needed with larger study populations pertaining specifically to OSA and COVID-19 patients.

\section{Acknowledgments}

None

\section{Conflicts of Interest}

The authors have no potential conflicts of interest to disclose.

\section{Author Contributions}

Conceptualization: Hyeon Hui Kang. Data curation: all authors. Formal analysis: Hyeon Hui Kang. Investigation: Hyeon Hui Kang. Methodology: Hyeon Hui Kang. Project administration: Hyeon Hui Kang. Resources: all authors. Supervision: all authors. Visualization: Hyeon Hui Kang, Writing—original draft: Hyeon Hui Kang. Writing—review \& editing: all authors.

\author{
ORCID iDs \\ Hyeon Hui Kang (D) \\ https://orcid.org/0000-0001-5860-9407 \\ Jin Hyoung Kim (D) \\ https://orcid.org/0000-0002-2229-2388 \\ Byung Ju Kang \\ https://orcid.org/0000-0002-1396-7398 \\ Taehoon Lee (D) \\ https://orcid.org/0000-0002-9224-0866 \\ Seung Won $\mathrm{Ra}$ (iD \\ https://orcid.org/0000-0002-2458-8414 \\ Kwang Won Seo (ID) \\ https://orcid.org/0000-0003-0504-6924 \\ Yangjin Jegal (D) \\ https://orcid.org/0000-0002-1935-7240 \\ Jong Joon Ahn (D) \\ https://orcid.org/0000-0002-1395-0801
}

\section{REFERENCES}

1. Zhou F, Yu T, Du R, Fan G, Liu Y, Liu Z, et al. Clinical course and risk factors for mortality of adult inpatients with COVID-19 in Wuhan, China: a retrospective cohort study. Lancet 2020;395:1054-1062.

2. Pazarlı AC, Ekiz T, İlik F. Coronavirus disease 2019 and obstructive sleep apnea syndrome. Sleep Breath 2021;25:371.

3. Tufik S, Gozal D, Ishikura IA, Pires GN, Andersen ML. Does obstructive sleep apnea lead to increased risk of COVID-19 infection and severity? J Clin Sleep Med 2020;16:1425-1426.
4. McSharry D, Malhotra A. Potential influences of obstructive sleep apnea and obesity on COVID-19 severity. J Clin Sleep Med 2020;16:1645.

5. Bhatraju PK, Ghassemieh BJ, Nichols M, Kim R, Jerome KR, Nalla AK, et al. Covid-19 in critically ill patients in the Seattle region-case series. N Engl J Med 2020;382:2012-2022.

6. Cade BE, Dashti HS, Hassan SM, Redline S, Karlson EW. Sleep apnea and COVID-19 mortality and hospitalization. Am J Respir Crit Care Med 2020; 202:1462-1464.

7. Strausz S, Kiiskinen T, Broberg M, Ruotsalainen S, Koskela J, Bachour A, et al. Sleep apnoea is a risk factor for severe COVID-19. BMJ Open Respir Res 2021;8:e000845

8. Oh TK, Song IA. Impact of coronavirus disease-2019 on chronic respiratory disease in South Korea: an NHIS COVID-19 database cohort study. BMC Pulm Med 2021;21:12.

9. Berry RB, Budhiraja R, Gottlieb DJ, Gozal D, Iber C, Kapur VK, et al. Rules for scoring respiratory events in sleep: update of the 2007 AASM manual for the scoring of sleep and associated events: deliberations of the sleep apnea definitions task force of the American Academy of Sleep Medicine. J Clin Sleep Med 2012;8:597-619.

10. Kushida CA, Chediak A, Berry RB, Brown LK, Gozal D, Iber C, et al. Clinical guidelines for the manual titration of positive airway pressure in patients with obstructive sleep apnea. J Clin Sleep Med 2008;4:157-171.

11. WHO Working Group on the Clinical Characterisation and Management of COVID-19 infection. A minimal common outcome measure set for COVID-19 clinical research. Lancet Infect Dis 2020;20:e192-e197.

12. Cariou B, Hadjadj S, Wargny M, Pichelin M, Al-Salameh A, Allix I, et al. Phenotypic characteristics and prognosis of inpatients with COVID-19 and diabetes: the CORONADO study. Diabetologia 2020;63:1500-1515.

13. Perger E, Soranna D, Pengo M, Meriggi P, Lombardi C, Parati G. Sleep-disordered breathing among hospitalized patients with COVID-19. Am J Respir Crit Care Med 2021;203:239-241.

14. Rouatbi S, Ghannouchi I, Kammoun R, Ben Saad H. The ventilatory and diffusion dysfunctions in obese patients with and without obstructive sleep apnea-hypopnea syndrome. J Obes 2020;2020:8075482.

15. Su VY, Liu CJ, Wang HK, Wu LA, Chang SC, Perng DW, et al. Sleep apnea and risk of pneumonia: a nationwide population-based study. CMAJ 2014; 186:415-421.

16. Memtsoudis SG, Ivascu NS, Pryor KO, Goldstein PA. Obesity as a risk factor for poor outcome in COVID-19-induced lung injury: the potential role of undiagnosed obstructive sleep apnoea. Br J Anaesth 2020;125:e262-e263.

17. American Academy of Sleep Medicine. Summary of CDC recommendations relevant for sleep practices during COVID-19 [Internet]. Available at: https://aasm.org/covid-19-resources/covid-19-mitigation-strategies-sleepclinics-labs/. Accessed April 8, 2020.

18. Zhang XL, Xiao Y. Sleep health service in China during the coronavirus disease outbreak. J Clin Sleep Med 2020;7:1221-1222.

19. Association for Respiratory Technology and Physiology. ARTP guidance respiratory function testing and sleep services during endemic COVID-19 [Internet]. Available at: https://www.artp.org.uk/News/artp-guidance-respiratory-function-testing-and-sleep-services-during-endemic-covid-19-august-2020. Accessed August 24, 2020.

20. Irish Thoracic Society-Irish Sleep Society. Guidance on Sleep/Non-invasive ventilation (NIV) services and CPAP/Domiciliary NIV use for obstructive sleep apnoea (OSA) OR obesity hypoventilation syndrome (OHS): SARS COVID-19 infection [Internet]. Available at: https://irishthoracicsociety.com/wpcontent/uploads/2020/03/ISSITS-Sleep-Guidelines-EM-V3b-002.pdf. Accessed March 15, 2020.

21. Ayas NT, Fraser KL, Giannouli E, Hanly PJ, Kendzerska T, Katz SL, et al. Helping Canadian health care providers to optimize sleep disordered breathing management for their patients during the COVID-19 pandemic. Can J Respir Crit Care Sleep Med 2020;4:81-82. 